Research Article

\title{
On connectivity of basis graph of splitting matroids
}

\author{
M. Pourbaba' ${ }^{1}$ (I) H. Azanchiler ${ }^{1}$
}

(c) Springer Nature Switzerland AG 2019

\begin{abstract}
For a set $C$ of circuits of a matroid $M, G(B(M), C)$ is defined by the graph with one vertex for each basis of $M$, in which two basis $B_{1}$ and $B_{2}$ are adjacent if $B_{1} \cup B_{2}$ contains exactly one circuit and this circuit lies in $C$. For two elements of $a$ and $b$ of ground set of a binary matroid $M$ a splitting matroid $M_{a, b}$ is constructed. It is specified by two collections of circuits $\mathcal{C}_{0}$ and $\mathcal{C}_{1}$ dependent with collections of circuits of $M$. We want to study connectivity of $G\left(B\left(M_{a, b}\right), \mathcal{C}_{0}\right)$ and $G\left(B\left(M_{a, b}\right), \mathcal{C}_{1}\right)$.
\end{abstract}

Keywords Basis graph · Splitting matroid · Connected matroid

\section{Introduction}

We assume that reader is familiar with the basic concepts of matroid theory and graph theory. For more details one can see [6] for matroid theory and [3] for graph theory. By considering bases of a matroid $M$, the basis graph of a matroid $M, G(B(M))$, is a graph in which each vertex is labeled as a basis of $M$ and two bases (vertices) $B_{1}$ and $B_{2}$ are adjacent if $\left|B_{1} \triangle B_{2}\right|=2$. In other words, two bases are adjacent if they differ in only one element. In [4] Holzmann and Harary proved that $G(B(M))$ is hamiltonian and therefore connected.

Many different variations of the basis graph have been studied, for instance, Li et al. [5] defined the basis graph of a matroid $M$ that related to a set $C$ of circuits. It is a spanning subgraph of $G(B(M))$ such that two bases $B_{1}$ and $B_{2}$ are adjacent if they are adjacent in $G(B(M))$ and the unique circuit of $M$ contained in $B_{1} \cup B_{2}$ is a circuit of $C$. In an special case when $M$ is a binary matroid, they found a sufficient condition and a necessary condition for this graph to be connected and they proved that given an element $e$ of a binary matroid $M$ and by supposing $C_{e}$ is the family of all the circuits that contains $e, G\left(B(M), C_{e}\right)$ is connected. Figueroa et al. [1] generalized this result and they proved that it is true for every matroid of $M$ with given $C_{e}$.
The splitting operation is defined for both graph and binary matroid. Fleischner [2] defined splitting operation for graph by the following way; let $G$ be a connected graph and suppose that $v$ is a vertex with degree at least 3 . Let $a=v v_{1}$ and $b=v v_{2}$ be two edges incident at $v$, then splitting away of $a, b$ from $v$ results in a new graph $G_{a, b}$ obtained from $G$ by deleting the edges $a$ and $b$, and adding a new vertex $v^{\prime}$ adjacent to $v_{1}$ and $v_{2}$. The transition from $G$ to $G_{a, b}$ is called the splitting operation on $G$. We also denote the new edges $v^{\prime} v_{1}$ and $v^{\prime} v_{2}$ in $G_{a, b}$ by $a$ and $b$, respectively.

The notion of the splitting operation extends to binary matroid in the following way [7]. Let $M=(E, C)$ be a binary matroid and $a, b$ be two elements of $E$. Let

$$
\begin{aligned}
\mathcal{C}_{0}= & \{C \in \mathcal{C}: a, b \in C \text { or } a, b \notin C\} \\
\mathcal{C}_{1}= & \left\{C_{1} \cup C_{2}: C_{1}, C_{2} \in C_{1} C_{1} \cap C_{2}=\emptyset,\right. \\
& a \in C_{1}, b \in C_{2} \text { and } C_{1} \cup C_{2} \\
& \text { contains no member of } \left.C_{0}\right\} .
\end{aligned}
$$

Let $\mathcal{C}^{\prime}=\mathcal{C}_{0} \cup \mathcal{C}_{1}$, then $M_{a, b}=\left(E, \mathcal{C}^{\prime}\right)$ is a binary matroid. As the collection of cycles of splitting graph is the same with the collection of circuits of splitting binary matroid defined above, we used the same notation. In [7], the authors showed that $M_{a, b}$ obtains from $M$ by adding an extra row to

M.Pourbaba, m.pourbaba@urmia.ac.ir; H. Azanchiler, h.azanchiler@urmia.ac.ir|'Department of Mathematics, Faculty of Science, Urmia University, Urmia, Iran.

SN Applied Sciences (2019) 1:444 | https://doi.org/10.1007/s42452-019-0470-z

Received: 11 January 2019 / Accepted: 8 April 2019 / Published online: 11 April 2019 
binary matrix representation of $M$, in which arrays respect to $a, b$ are 1 and remind arrays are 0 .

Shikare and Azadi [8] characterized the collection of bases of a splitting binary matroid as the following theorem.

Theorem 1.1 Let $M=(E, C)$ be a binary matroid and $a, b \in E$. Let $B$ be the set of all bases of $M$. Let $\mathcal{B}_{a, b}=\{B \cup\{\alpha\}: B \in \mathcal{B}, \alpha \in E-B$ and the unique circuit contained in $B \cup\{\alpha\}$ contains either $a$ or $b\}$. Then $B_{a, b}$ is $a$ set of bases of $M_{a, b}$.

\section{Main results}

We shall want to consider two cases of $G\left(B\left(M_{a, b}\right), C\right)$ respect the two collections of circuits of $M_{a, b}$. First, we start with a helpful lemma.

Lemma 2.1 Let $M$ be a binary matroid and $T=\{a, b, c\}$ be a triangle of $M$. Let $B_{1}$ be a basis of $M$ contains two elements of $T$. Let $B_{2} \subseteq E(M)$ such that $\left|B_{2}\right|=\left|B_{1}\right|$ and $B_{2}-B_{1}=\{e\}$ where $\in T$, then $B_{2}$ is a basis of $M$.

Proof Let $B_{1}=\{a, b, 1,2, \ldots, n\}$ be a basis of $M$ and let $B_{2}=\{a, t, 1,2, \ldots, n\}$ be the assumed subset of the lemma. It is clear that $X=\{a, 1,2, \ldots, n\}$ is independent, we prove $X \cup\{t\}=B_{2}$ is too. Assume the contrary and let $X \cup\{t\}$ contains a circuit $C$. Clearly $t \in C$. If $a \in C$, as $M$ is binary and $\{a, b, t\}$ is a triangle, then $a+b=t$. So $C^{\prime}=b \cup C-\{t, a\}$ is a circuit and $C^{\prime} \subseteq B_{1}$, a contradiction. If $a \notin C$, then $C^{\prime \prime}=\{a, b\} \cup C-\{t\}$ is a circuit and $C^{\prime \prime} \subseteq B_{1}$, a contradiction. Thus $X \cup\{t\}$ is independent and therefore $B_{2}$ is a basis of $M$.

The following theorem is our main result.

Theorem 2.2 Let $M$ be a binary matroid and $T=\{a, b\}$, where $a, b \in E(M)$. Let $C_{0}$ be the collection of circuits in $M$ in which meet $T$ at even elements, then a sufficient condition for $G\left(B\left(M_{a, b}\right), C_{0}\right)$ to be connected is that $T$ lies in a triangle in $M$.

Proof If $C_{1}$ at the collection of circuits of splitting matroids is empty set, then $M_{a, b}=M$ and hence $C_{0}=C(M)$. Therefore $G\left(B\left(M_{a, b}\right), \mathcal{C}_{0}\right)=G(B(M))$ and $G(B(M))$ always is connected. Thus, suppose $C_{1}$ is non-empty set. Let $\{a, b, t\}$ be the assumed triangle. We consider four cases about vertices of $G\left(B\left(M_{a, b}\right), C_{0}\right)$. In these cases $B_{i}$ are bases of $M_{a, b}$ as characterized in Theorem 1.1 and $x, y \in E\left(M_{a, b}\right)-T$.

Case $1 B_{1}=\{a, 1,2, \ldots, x\}$ and $B_{2}=\{a, 1,2, \ldots, y\}$.

Since $M_{a, b}$ does not have a circuit with just an element of $T$, then $B_{1} \cup B_{2}$ contains a circuit $C$ that avoids $a$. Hence
$C \cap T=\emptyset$, so $C \in \mathcal{C}_{0}$. We conclude $B_{1}$ and $B_{2}$ are adjacent in $G\left(B\left(M_{a, b}\right), C_{0}\right)$.

Case $2 B_{1}=\{a, 1,2, \ldots, x\}$ and $B_{2}=\{a, 1,2, \ldots, b\}$.

Suppose $B_{1}$ and $B_{2}$ are not adjacent. Then $t \notin B_{1} \cup B_{2}$; otherwise the triangle $\{a, b, t\}$ contained in $B_{1} \cup B_{2}$, contradicting the fact that $B_{1}$ and $B_{2}$ are not adjacent. By Lemma 2.1 $B_{3}=\{a, 1,2, \ldots, t\}$ is a basis of $M_{a, b}$. Now $B_{2} \cup B_{3}$ contains a unique circuit in which $t$ and $b$ belong to it and since $a$ belongs to that union, the circuit is the triangle. Thus, it is on $C_{0}$. Then $B_{2}$ and $B_{3}$ are adjacent. By the first case $B_{1}$ and $B_{3}$ are adjacent. Then there is a path from $B_{1}$ to $B_{2}$.

Case $3 B_{1}=\{a, b, 1,2, \ldots, x\}$ and $B_{2}=\{a, b, 1,2, \ldots, y\}$.

If a circuit $X$ in $B_{1} \cup B_{2}$ is a member of $C_{0}$, the result is trivial and this two vertices are adjacent in $G\left(B\left(M_{a, b}\right), C_{0}\right)$. By the Lemma 2.1, $B_{3}=\{a, t, 1,2, \ldots, x\}$ and $B_{4}=\{a, t, 1,2, \ldots, y\}$ are two bases of $M_{a, b}$. By the second case $B_{1}=\{a, b, 1,2, \ldots, x\}$ and $B_{3}=\{a, t, 1,2, \ldots, x\}$ are adjacent and similarly two bases of $B_{2}=\{a, b, 1,2, \ldots, y\}$ and $B_{4}=\{a, t, 1,2, \ldots, y\}$ are adjacent by the second case. In the other hand $B_{3}$ and $B_{4}$ are adjacent by the first case. Therefore there is a path between $B_{1}$ and $B_{2}$.

Case $4 B_{1}=\{1,2, \ldots, a\}$ and $B_{2}=\{1,2, \ldots, b\}$.

Suppose $B_{1}$ and $B_{2}$ are not adjacent in $G\left(B\left(M_{a, b}\right), C_{0}\right)$. The uniqe circuit $X$ in $B_{1} \cup B_{2}$ is disjoint union of two circuits $C_{1}$ and $C_{2}$ of $M$ such that each of them meets $T$ precisely in one element. In fact $C_{1}=\left\{a, a_{1}, a_{2}, \ldots, a_{m}\right\} \subseteq B_{1}$ and $C_{2}=\left\{b, b_{1}, b_{2}, \ldots, b_{n}\right\} \subseteq B_{2}$. It is clear that $t \notin B_{1} \cup B_{2}$. We construct $B_{3}$ by the following way; we delete a member of $C_{1}$ like $a_{1}$ and add $t$ in it. Without loss of generality, we can assume that $a_{1}=1$. Hence $B_{3}=\{t, 2, \ldots, a\}$. Since $a+b=t$ and $B_{3}-\{a, t\} \subseteq B_{2}$ it is clear that $B_{3}$ is a basis of $M_{a, b}$. Now by the second case $B_{1}$ and $B_{3}$ are connected by a path. Suppose $B_{4}=\{b, 2, \ldots, a\}$ is constructed by deleting $t$ and adding $b$. By the Lemma 2.1, $B_{4}$ is a basis of $M_{a, b}$. By the second case $B_{4}$ and $B_{3}$ are connected by a path too. Now if we apply the same procedure for the basis $B_{2}$ and without loss of generality by considering $b_{1}=2$, we get bases $B_{5}=\{1, t, \ldots, b\}$ and $B_{6}=\{1, a, \ldots, b\}$ that by the second case there is a path between $B_{2}$ to $B_{5}$ and a path between $B_{5}$ to $B_{6}$. Now notice that $B_{4} \triangle B_{6}=\{1,2\}$ and $B_{4}$ and $B_{6}$ are connected by a path by the second case. Hence there is a path between $B_{1}$ and $B_{2}$.

Now suppose that $B_{1}$ and $B_{2}$ are two arbitrary bases of $M_{a, b}$. As $G\left(B\left(M_{a, b}\right)\right)$ is connected, there is a path from $B_{1}$ to $B_{2}$ in $G\left(B\left(M_{a, b}\right)\right)$. Then $B_{1}$ has an adjacent vertex in $G\left(B\left(M_{a, b}\right)\right)$. This two vertices are connected with a path by using four cases mentioned above. Thus we conclude $B_{1}$ and $B_{2}$ in $G\left(B\left(M_{a, b}\right), C_{0}\right)$ are connected by a path, then the graph $G\left(B\left(M_{a, b}\right), C\right)$ is connected.

Evidently the sufficient condition in the theorem is not necessary. Consider the following example. 
Example 2.3 For the graph $G$ in the following Figure (a) $G_{a, b}$ is shown in Figure (b). There is no triangle in $G$ but one can show $G\left(B\left(M(G)_{a, b}\right), C_{0}\right)$ is connected.

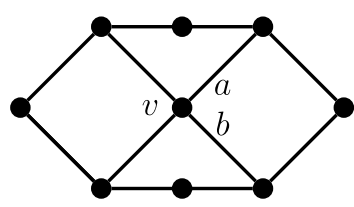

(a) $G$

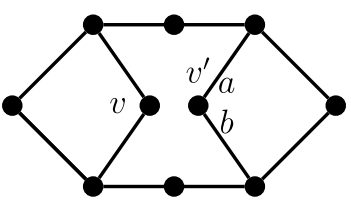

(b) $G_{a, b}$
Corollary 2.4 Let $M$ be a matroid and $C_{0}$ be specified collection of circuits in the last Theorem. Let $S$ be a triangle of $M$. Suppose $T=\{a, b\}$ and $T, S$ are disjoint. If $M_{x, y}=M_{a, b}$, where $x, y \in S$, then $G\left(B(M), C_{0}\right)$ is connected.

Theorem 2.5 Let $a$ and $b$ be two elements of $a$ binary matroid $M$. If $\mathcal{C}_{0}$ is empty, then $G\left(B\left(M_{a, b}\right), \mathcal{C}_{1}\right)$ is connected.

Proof Suppose $\mathcal{C}_{0}$ is empty. If $\mathcal{C}_{1}$ is empty, then $M_{a, b}$ is a free matroid and clearly $G\left(B\left(M_{a, b}\right), C_{1}\right)$ is connected. Thus, we can assume that $C_{1}$ is non-empty. Now suppose $C$ and $C^{\prime}$ are two circuit of $M_{a, b}$. As $C_{0}$ is empty, so $a$ and $b$ belong to both of $C$ and $C^{\prime}$. Since $M_{a, b}$ is binary then $C \triangle C^{\prime}$ contains a circuit and none of $a$ or $b$ belongs to this circuit, hence $\mathcal{C}_{0}$ is non-empty, a contradiction. Thus $M_{a, b}$ just has one circuit. Therefore $G\left(B\left(M_{a, b}\right), C_{1}\right)$ is a complete graph and hence it is connected.

Notice that the converse of the last theorem is not true generally, for instance consider following example.

Example 2.6 Consider the graph $G$ and its splitting graph $G_{a, b}$ in following figure. One can easily check that there is a path between every two vertices of $B\left(M(G)_{a, b}, C_{1}\right)$. Hence $B\left(M(G)_{a, b}, C_{1}\right)$ is connected while $\mathcal{C}_{0}$ is non-empty.



G

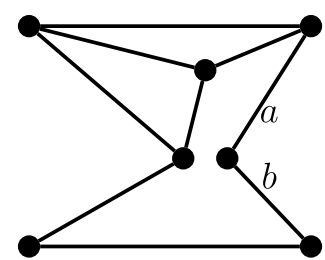

$G_{a, b}$
The converse of Theorem 2.5 can be true with a special condition.

Theorem 2.7 Let $a$ and $b$ be two elements of a binary matroid $M$. Let $C_{0}$ be non-empty and $\mathcal{C}_{1}$ has only one circuit. Then $G\left(B\left(M_{a, b}\right), C_{1}\right)$ is disconnected.

Proof Let $C_{1}$ be the only circuit of $\mathcal{C}_{1}$. Since $\mathcal{C}_{0}$ is non-empty, there is a basis of $M_{a, b}$ called $B_{1}$ such that

$\left|C_{1}-B_{1}\right| \geq 2$.

Let $B_{2}$ be a basis of $M_{a, b}$ in which contains $C_{1}-e$, where $e \in C_{1}$. We claim that vertices $B_{1}$ and $B_{2}$ of $G\left(B\left(M_{a, b}\right), C_{1}\right)$ are not adjacent. Assume the contrary, let $B_{1} \cup B_{2}$ contains a circuit of $C_{1}$, that is $C_{1}$. As $B_{2}=\left(B_{1}-f\right) \cup g$, where $f \in B_{1}$ and $g \in B_{2}$, by $(1),\left|C_{1}-\left(B_{1} \cup B_{2}\right)\right| \geq 1$. Hence $C_{1}$ is not contained in $B_{1} \cup B_{2}$, contradiction. In fact $B_{1}$ has no adjacent vertex, then $G\left(B\left(M_{a, b}\right), C_{1}\right)$ is disconnected.

Acknowledgements The authors gratefully acknowledge the Faculty of Science of Urmia University for helpfull support given.

\section{Compliance with ethical standards}

Conflict of interest The authors declare that they have no conflict of interest.

\section{References}

1. Figueroa AP, Campo E (2012) The basis graph of a bicolored matroid. Discret Appl Math 160:2694-2697

2. Fleischner H (1990) Eulerian graphs and related topics: part 1, vol 1. North-Holland, Amsterdam

3. Harary F (1969) Graph theory. Addison-Wesley, Boston

4. Holzmann CA, Harary F (1972) On the tree graph of a matroid. SIAM J Appl Math 22:187-193

5. Li X, Neumann-Lara V, Rivera-Campo E (2003) The tree graph defined by a set of cycles. Discret Math 271:303-310

6. Oxely JG (2011) Matroid theory, 2nd edn. Oxford University Press, New York

7. Raghunathan TT, Shikare MM, Waphare BN (1998) Splitting in a binary matroid. Discret Math 184:267-271

8. Shikare MM, Azadi Gh (2003) Determination of the bases of a splitting matroid. Eur J Comb 24:45-52

Publisher's Note Springer Nature remains neutral with regard to jurisdictional claims in published maps and institutional affiliations. 